\title{
El Derecho Penitenciario en el currículo formativo de los Abogaros en el
} Ecuador

\section{Penitentiary Law in the training curriculum of Lawyers in Ecuador}

\author{
Luis Andrés Crespo-Berti \\ ui.luiscrespo@uniandes.edu.ec \\ Universidad Regional Autónoma de los Andes, Ibarra \\ Ecuador \\ https://orcid.org/0000-0001-8609-4738 \\ Jorge Luis Nogales-Tello \\ di.jorgelnt09@uniandes.edu.ec \\ Universidad Regional Autónoma de los Andes, Ibarra \\ Ecuador \\ https://orcid.org/0000-0001-7170-1718 \\ Carlos Steven Mediavilla-Flores \\ di.carlossmf66@uniandes.edu.ec \\ Universidad Regional Autónoma de los Andes, Ibarra \\ Ecuador \\ https://orcid.org/0000-0003-2797-4465
}

Recepción: 15 de septiembre 2021

Revisado: 25 octubre 2021

Aprobación: 15 de noviembre 2021

Publicación: 01 de diciembre 2021 


\title{
RESUMEN
}

El objetivo de esta investigación se centró en analizar el derecho penitenciario en el currículo formativo de los abogaros en el Ecuador. Apoyado en el enfoque cuantitativo, sumido al análisis inductivo, sistemático, crítico, bibliográfico-documental y de análisis de contenido en atención al modelo de investigación de tipo exploratoria, en el afán de los autores por generar un conjunto de conocimientos fundamentados en la teoría del objeto de estudio, considerando la dinámica social y la capacidad de establecer teorías que enriquezcan la investigación en la comunidad jurídica. El diseño bibliográfico en apoyo del análisis de contenido, responde a la revisión de fuentes primarias de información, las mallas curriculares y el plan de estudio universitario. Al concluir que la instrucción (pedagogía) del Derecho Penitenciario como asignatura autónoma e independiente, corresponde a un compromiso mejor idealizado con la sociedad. De manera que, el estudiante graduado ayude a mejorar el comportamiento de la ciudadanía.

Descriptores: Educación superior; derecho; ciencias de la educación; enseñanza; justicia. (Palabras tomadas de Tesauro UNESCO).

\begin{abstract}
The objective of this research was focused on analyzing penitentiary law in the training curriculum of lawyers in Ecuador. Supported by the quantitative approach, submerged in inductive, systematic, critical, bibliographic-documentary analysis and content analysis in attention to the exploratory research model, in the authors' desire to generate a set of knowledge based on the theory of object of study, considering social dynamics and the ability to establish theories that enrich research in the legal community. The bibliographic design in support of the content analysis, responds to the review of primary sources of information, the curricular meshes and the university study plan. Concluding that the instruction (pedagogy) of Penitentiary Law as an autonomous and independent subject, corresponds to a better idealized commitment to society. Thus, the graduate student helps improve the behavior of citizens.
\end{abstract}

Descriptors: Higher education; right; educational sciences; teaching; Justice. (words takenfrom UNESCO Thesaurus).

\section{INTRODUCCIÓN}


En el transcurso de la formación del profesional de Derecho, el estudiante en cuestión presenta una serie de incógnitas que se presentan a modo de curiosidad, todo esto referente al futuro académico de instrucción y enseñanza. Desde que el estudiante de Derecho decide formar parte de esta carrera universitaria se interesa por conocer cuáles asignaturas formaran parte de su malla curricular, y una vez tomada la decisión se entusiasma por el futuro de aprender. Es entonces, que se infiere que las asignaturas tienen una importancia muy significativa para los estudiantes, ya que de ellas dependen en el cómo el futuro profesional de Derecho se desenvuelva según su gusto o preferencia dentro de las diversas especialidades del Derecho.

Pero, en un contexto de formación; el estudiante se siente curioso por conocer las asignaturas cada vez que escala niveles en la carrera de Derecho, y de esta curiosidad nace el problema de los autores para esta investigación: ¿Por qué no se instruye el Derecho Penitenciario, en la malla curricular? La necesidad de tener una formación más especializada hace que se plantee las bondades que tiene esta asignatura en su posible instrucción, ya que se presenta como una asignatura olvidada que tan solo entra en un porcentaje menor dentro del todo de la materia de Derecho Procesal.

En este sentido, la calidad de las universidades responde de manera directa a la estructuración de las mallas curriculares, plantel docente, estudiantes, entre otros. En el caso del Ecuador la Ley Orgánica de Educación Superior dispone (LOES, 2010) las instituciones que rigen la educación superior en el país, se han realizado intentos para garantizar dicha calidad, para fomentar la vinculación con la sociedad y la investigación en busca del desarrollo de la matriz cognitiva (Castellanos, 2019, p.11). Gracias al precedente y sentido de pertinencia los estudiantes apoyados de sus derechos, pueden solicitar una mejora al sistema educativo.

Dado la pertinencia, se manifiesta la necesidad del Derecho Penitenciario en las carreras de Derecho a nivel nacional. Dicho esto, es importante denotar de manera general el significado del Derecho Penitenciario: "Conjunto de normas que regulan la ejecución de las penas y medidas penales privativas de libertad y de la pena de trabajos en beneficio de la comunidad." (RAE, 2021). Inmerso en la formación del profesional del derecho, tiene por excelencia dos ramas del Derecho de suma importancia que son el Derecho Civil y el Derecho Penal, además eventualmente 
inserto en las Ciencias procesales. De las dos ramas presentes la que es pertinente de la investigación es el Derecho Penal, del cual es el que se deriva el Derecho Penitenciario.

No obstante, el Derecho Penitenciario se erige como un puntual que erróneamente cae en el paradigma del fin del proceso penal, y al contrario es un apartado independiente y autónomo muy importante, pues asegura las garantías que tienen las personas privadas de libertad una vez la sentencia se encuentre ejecutoriada, la realidad indica que el debido proceso se termina con la sentencia; pero al contrario, se infiere que empieza una nueva etapa a la que se denomina proceso penitenciario, en el cual entra el Derecho como tal, la realidad del Ecuador hace que una vez la sentencia esté ejecutoriada el sentenciado sea prácticamente olvidado dentro de la ley, a pesar de gozar con garantías constitucionales. Es decir, que la falta de especialización en esta área hace que la persona privada de libertad sea vulnerable a cualquier violación de derechos constitucionales e incluso humanos, por ende, si el profesional del Derecho está capacitado tenga la suficiente razón y argumento para salvaguardar la integridad de la persona privada de libertad.

Entre tanto, Coca (2007), sostiene que el tratamiento penitenciario hasta hace algunos años no se discutía y se consideraba un magnífico avance progresista dentro de un contexto de humanización de las prisiones (p.170). Lamentablemente dicha humanización está muy lejos de ser real ya sea en el contexto ecuatoriano como latinoamericano.

Por tanta, la razón de la investigación se nutre de manera conveniente para los autores, con la realidad reciente de los centros de rehabilitación social en el Ecuador, en el cual la situación es bastante preocupante si no ya se ha salido de las manos, y eso se da por una falta de aplicación correcta del Derecho Penitenciario, que evidentemente esta investigación no expone a la falta de instrucción del Derecho Penitenciario en las carreras de Derecho como el principal responsable de la situación que se vive, sino que es parte de un conjunto de negligencias que hace que se vulnere derechos.

El derecho penitenciario se inserta en la sociedad como una ciencia, tal como el derecho penal. Así el estudio del derecho penal supone las normas penales relativas 
a un Estado, también la necesidad de controlar el ius puniendi Estatal ejercido desde el ámbito legislativo hasta las ramas de la fuerza pública, pasando por la función judicial como titular de la misión de juzgar (Zaffaroni, 2009). Por analogía el derecho penitenciario surge a partir de establecer un conjunto de conocimientos que refieran establecer criterios doctrinarios en el afán de configurar la normativa de ejecución penal en estricta sujeción a los derechos fundamentales que aún conserva aquel sujeto sancionado con la pena y que espera el fin de la misma en un sistema de ejecución que surge a partir de una sentencia.

Así, Durán (2020), señala que, el derecho penitenciario trata sobre la ejecución de las penas, una rama de las ciencias jurídicas que se encarga del estudio de las reglas relativas al inicio y cumplimiento de las penas, medidas de seguridad impuestas como consecuencia de un delito, además, trata los derechos de aquellos sujetos impuestos a una pena. Puesto que, dado el enfoque normativo de aplicación de los derechos humanos, supone de un conjunto de reglas de aplicación de normas ejecutivas en materia penitenciaria y a la par un conjunto de garantías que se le atribuyen no solo al privado de la libertad, sino también a aquellos sujetos a penas diferentes.

Por otro lado, es importante concebir que el derecho penitenciario contiene las normas materiales y formales para la ejecución de sanciones penales, dado el sentido que configura un sistema que regula las medidas privativas de libertad o aquellas que implican ser parte de la privación de libertad. En consecuencia, se torna inevitable concebir a la ejecución penal como una materia homogénea (Duran, 2020). Es este sentido es importante considerarla homogénea e individual siempre que se debe considerar la finalidad del cumplimiento de la pena, al ser elemento indiscutible de la ejecución penal. La pena debe tener un fin, no es de cumplir el tiempo establecido en la sanción, si no el fin resocializador (Durán y Prado, 2020). En este sentido, la resocialización puede lograrse al amparo del respeto a los derechos fundaméntales por parte de un sistema de ejecución penal organizado a respecto del respeto a los beneficios penitenciarios, toda vez que aquellos a quienes se les ha impuesto una pena resultan ciudadanos a los ojos de un Estado de Derecho.

Con base en los argumentos establecidos con anterioridad, es necesario identificar una variable de transcendental importancia al referir al derecho penitenciario como 
una disciplina autónoma deslindada de la teoría de proceso penal, esto es, señalarla dentro los sistemas de educación superior como una ciencia homogénea que desprende por si sola la cantidad de conocimientos necesarios en el ámbito de ejecución penal para el estudiante de Derecho. ¿Es importante considerar al derecho penitenciario como materia homogénea en la carrera de Derecho?

Al respecto es menester establecer la importancia de un correcto desarrollo pedagógico en las ciencias jurídicas, toda vez que la fundamentación teórica en las aulas de la educación superior, supone un cambio en la sociedad. Según Zambrano (2017), la pedagogía es la teoría práctica de la educación (Citado en Garzón y Romero, 2017. p. 313). Un sistema que genera conocimientos a partir de la realidad actual, con proyección a mejorar la sociedad con respecto los cambios que se puedan generar respecto a una materia específica.

Por otro lado, el iusnaturalismo asume el respeto a la dignidad humana que surge a partir del derecho natural. En ese sentido Ferrajoli (2009), apunta a los derechos fundamentales como una construcción jurídica de derechos universales a todos los seres humanos, normas previstas de carácter positivo al estar inmersas en un cuerpo legal. De cierto modo, la dignidad humana prevista por el iusnaturalismo se entrelaza a manera de evolución a la norma positiva, toda vez que se vuelve de estricto cumplimiento con el afán de proteger al ciudadano.

En este sentido, se vuelve prioridad contener la conservación de la dignidad y la sujeción a la ley en el ámbito del derecho penitenciario, puesto que, según lo expuesto anteriormente; el derecho penitenciario no solo reúne aptitud de verificar el cumplimiento de la pena, en este caso, refiere también a los derechos que el condenado posee, así como a la finalidad de reinserción social que contempla para el mismo. En consecuencia, la humanización de la vida humana debe estar presente en el proceso de formación de un estudiante de derecho, por lo tanto, en la pedagogía en el derecho no debe partir desde una posición absolutista en referencia al objeto de estudio.

En el Ecuador se concibe al derecho penitenciario como un tema novísimo en el sentido estricto, puesto que es contemplado desde el punto de vista procesal penal. Entonces, se deja de lado la homogenización de la materia en cuestión. Además, el 
incremento de la sensibilidad popular ante el cometimiento de una infracción ha generado que el castigo o pena se vincule directamente a la cultura de la sociedad, por aquello de que la privación de libertad se convierte en una necesidad y solución a un problema social que apunta a los estratos sociales más bajos. La cárcel y el sistema penitenciario suponen un instrumento de miseria y dolor, donde el fin es el castigo y no la reinserción (Vargas, 2016). Se aleja de todo lo establecido por los preceptos del derecho penitenciario, esa es la realidad, donde el Estado olvida a los condenados a causa del resentimiento de la sociedad; desprecio notable hasta en sus sistemas de educación superior donde el derecho penitenciario es visto como secundario a pesar de que los condenados deben ser de atención prioritaria a la luz de un Estado social de Derechos.

La Constitución de la República del Ecuador (2008), en el artículo 47, los resalta como miembros de atención prioritaria y sujetos de derechos fundamentales descritos en este cuerpo legal y en los tratados internacionales, siempre que pierden los derechos sujetos a la pena impuesta; como los derechos políticos en el caso de existir una sentencia ejecutoriada. En este sentido, se vuelve indispensable la mejora de un sistema penitenciario con el fin de establecer un sistema ejecutivo que se ejecute en estricto apego a las necesidades humanas de aquellos impuestos una pena de privación de libertad (Durán y Prado, 2020).

El objetivo de esta investigación se centra en analizar el derecho penitenciario en el currículo formativo de los abogaros en el Ecuador.

\section{METODOLOGÍA}

La presente entrega investigativa se sustenta en el enfoque cuantitativo, sumido al análisis inductivo, sistemático, crítico, bibliográfico-documental y de análisis de contenido en atención al modelo de investigación de tipo exploratoria, en el afán de los autores por generar un conjunto de conocimientos fundamentados en la teoría del objeto de estudio, considerando la dinámica social y la capacidad de establecer teorías que enriquezcan la investigación en la comunidad jurídica. De este modo, el 
método de comparación constante, sirve como modelo de corte y clasificación al momento de procesar la información obtenida del objeto de estudio (Bonilla y López, 2016). Así mismo, el diseño bibliográfico en apoyo del análisis de contenido, responde a la revisión de fuentes primarias de información, toda vez que se suponen fidedignas al respecto de la investigación científica, en esto, las mallas curriculares y el plan de estudio de las diferentes universidades locales y extranjeras, la doctrina relacionada con el derecho penitenciario, artículos científicos referentes a los dilemas que emergen de la concepción del derecho penitenciario; sirven de herramienta en base a un análisis sistemático, riguroso y profundo para establecer el estado de la cuestión y fundamentar la discusión de resultados. Creswell (1998) plantea que el análisis de estos datos se basa en la finalidad de cubrir facetas y ángulos del mismo fenómeno de estudio (Citado en Hernández, Fernández y Baptista, 2014).

\section{RESULTADOS}

\section{Participación del derecho penitenciario}

Es importante estudiar el Derecho Penitenciario desde el punto de vista holístico en referencia a la valoración de todo el material jurídico que se subsume a la materia, esto es, estudiar todos los elementos que hacen al derecho penitenciario en un sentido amplio que enriquezca de conocimientos a quien lo intenta analizar. De este modo es menester iniciar por establecer la definición desde el punto de vista doctrinario del Derecho Penitenciario que a rigor de Cesano (2007), refiere a un conjunto de normas que regulan las relaciones jurídicas derivadas de la ejecución de las penas privativas de libertad, así por sinonimia se subsume a la ejecución penal siempre que debe ser incluido el estudio de las penas diferentes a las privativas de libertad y las medidas de seguridad.

Desde lo expuesto, es importante acudir al establecimiento de la realidad del derecho penitenciario en el Ecuador, esto es, como se concibe el derecho penitenciario en la esfera local. En este sentido, el estudio de la materia se considera nulo, siempre que se aleja de la noción del derecho penitenciario y en el mejor de los casos se refiere a un sistema penitenciario. Por lo tanto, se infiere que la acogida de la temática expuesta es nula, toda vez que no se toma en cuenta como una materia autónoma desde el 
punto de vista doctrinario, si no que se torna una problemática al aludir una crisis en el sistema penitenciario. Esto, basado en el supuesto del desconocimiento de la amplitud de temas que nacen del Derecho Penitenciario.

Esta problemática es importante atribuirla a la práctica pedagógica de la materia en cuestión, toda vez que el derecho penitenciario se subsume al derecho procesal penal. En tanto que a pesar de que el Código Orgánico Integral Penal (2014), vigente a la fecha, se atribuye la facultad de establecer un libro entero en el que se detalla lo referido a la ejecución penal, esto es el libro tercero de la ley mencionada. A la par la Constitución de la República del Ecuador (2008), en sus artículos 35 y 51, alude a que las personas privadas de la libertad forman parte del grupo de atención prioritaria, por lo tanto el Estado se somete a prestar especial protección a estos grupos que a la luz del pensamiento popular se consideran enemigos de la sociedad, no obstante el cuerpo legal desarrolla un conjunto de derechos que, además de los fundamentales, son atribuidos a su persona, esto en estricto apego a los derechos que por motivo de condena son excluidos de los preceptos que se le atribuye al privado de la libertad. En esto, se considera pertinente establecer que la realidad apunta al olvido de estas cuestiones, toda vez que se refleja en la escasa, por no ser nula participación del Derecho Penitenciario en el ejercicio de la pedagogía en la educación superior.

\section{Necesidad de una pedagogía asociada al derecho penitenciario}

Silvera, Garcés y Pineda (2018), en referencia a la pedagogía, aseveran que el propósito del saber pedagógico se centra en incorporar en el quehacer del maestro un proceso integrador que fortalezca la enseñanza y el aprendizaje, toda vez que se pretenda generar un estado de tranquilidad frente a la satisfacción de las necesidades y aspiraciones de los participantes en el proceso educativo. Por lo tanto, no es indiferente la necesidad de potenciar el estudio del derecho en una rama específica como es la del Derecho Penitenciario. De ese modo la pedagogía se inserta como la necesidad de enseñar el tema referido y que a la vez esto genere un estado de 
fortaleza al estudiante, en virtud de los conocimientos adquiridos que a lo posterior sirven de mejora a la sociedad.

En las universidades ecuatorianas es nula la acogida del Derecho Penitenciario como asignatura autónoma. En el mismo sentido, las universidades argentinas no acogen al Derecho Penitenciario como asignatura en sus respectivos planes de Estudio. No obstante, Perú y México si lo hacen, en el sentido de que refieren unas como materia optativa y en otras como asignatura obligatoria. De aquello se desprende una notable deficiencia en cuanto a la inclusión diversificada en las materias de interés jurídico en el sistema educativo latinoamericano. A pesar que la educación superior en la región se ha constituido en avance en pro de lograr mejoras a los procesos pedagógicos, con respecto a que se ha logrado generar una conveniente orientación hacia una formación integral de los profesionales (Alpizar y Molina, 2017).

Así también es importante establecer que, en países como España e Italia, respecto a sus universidades y en específico a sus planes de estudio, la acogida de Derecho penitenciario es bastante amplia, a la luz que se contempla en gran medida como asignatura de nivel de obligatorio cumplimiento. De lo expuesto, a modo de opinión, el sistema de educación en derecho en Latinoamérica se muestra deficiente ante el sistema italiano, a pesar de que se presume cuna del derecho en el sistema latino, toda vez que se somete a las directrices establecidas por el Derecho Romano.

\section{Contexto nacional del Derecho Penitenciario}

Es incomprensible no considerar la crisis carcelaria, y los problemas que derivan de una ineficiente e ineficaz aplicación de lo establecido en términos de ejecución penal. La sociedad ecuatoriana se suma a una crisis inevitable en los sistemas penitenciarios. A la luz de los ciudadanos se visibiliza una notable falta de preocupación por los privados de la libertad, que se traduce en violación de sus derechos constitucionales y derechos humanos establecidos en los tratados internacionales. El hacinamiento y olvido genera que el sistema se derrumbe en el sentido de establecer condiciones adecuadas al privado de la libertad para un proceso de reinserción a la sociedad que asegure la protección del quebrantamiento de la 
norma por parte de los infractores. El sentimiento de venganza hace que se le otorgue otros fines a la pena, de lo establecido en la doctrina. Y la cárcel se vuelve la jungla donde sobrevive el más fuerte. Es obvio, si desde el punto de vista educativo en derecho, se olvida al régimen penitenciario y se lo intenta excluir del sistema de educación.

\section{Importancia de la instrucción del Derecho Penitenciario en la carrera de Derecho en el Ecuador}

La inclusión del Derecho Penitenciario en las mallas curriculares de la carrera de Derecho a nivel nacional, se presenta como necesaria e inmediata, puesto que, el análisis de las mallas curriculares de las principales universidades del Ecuador no toma al Derecho Penitenciario como una asignatura autónoma, al contrario se da todo el peso al Derecho Procesal Penal como materia madre, dejando escaso contenido para la cátedra del Derecho Penitenciario que en composición es una asignatura que en todo su constructo no le hace falta merecimientos o razones para ser autónoma. La correcta inclusión de esta asignatura en las mallas curriculares de la carrera de Derecho, garantizará una mejor formación del estudiante tanto en la variable humanista como profesional, puesto que la labor del abogado es netamente de ayuda y compromiso para la sociedad. Si la misión de la privación de la libertad es de reintegrar ciudadanos correctos a la sociedad, se debe garantizar el compromiso de cumplir las leyes, aquí el profesional de Derecho instruido con esta nueva cátedra será capaz de cumplir los preceptos constitucionales, para de esta manera garantizar la correcta corrección de los que se considera delincuentes. La educación cambia paradigmas, y crea sueños inspiradores.

\section{DISCUSIÓN}

En respuesta al planteamiento y de las posiciones encontradas, se determina que el Derecho Penitenciario se erige como una rama del Derecho especial y técnica; tanto en la forma de su aplicación como en la manera de instruir (pedagogía). El contexto del Estado ecuatoriano, en sus políticas de mejorar la educación superior hace que los propios involucrados como lo son estudiantes; tengan una participación más activa 
en cuanto a la calidad y calidez de la educación en las diferentes carreras que ofertan las universidades. En específico el punto de pertinencia, radica en el área de la carrera de Derecho a nivel nacional; no obstante, la necesidad de encontrar información a fin al objeto de controversia, se enriquece con la influencia de universidades foráneas con la intención de mejorar la investigación. Caso que se adjunta, a un problema identificado que es la falta de autonomía e independencia del Derecho Penitenciario como asignatura, tanto en Ecuador como en el mundo. Practicado una regresión, la mejora de la educación en el punto de injerencia de la investigación, precisa la incorporación del Derecho Penitenciario como asignatura en la carrera de Derecho a nivel nacional; en cuanto el estudiante propone su mejora a través de la indispensable investigación.

\section{CONCLUSIONES}

Contextualizando el estudio de la investigación y analizando de manera exhaustiva la discusión, se afirma que la instrucción (pedagogía) del Derecho Penitenciario, se presenta como necesaria e indispensable en las mallas curriculares de la carrera de Derecho a nivel nacional, a efectos que el futuro abogado tenga los conocimientos necesarios para ejercer su profesión, dicho esto, quienes suscriben la presente investigación llegan a las siguientes conclusiones:

Para lograr una correcta formación del profesional del derecho desde su alma mater, se necesita un análisis cualitativo, cuantitativo y crítico de las diferentes ramas del Derecho, relacionados con la pedagogía de las universidades que ofertan la carrera de Derecho. Dato, se infiere puesto que existen asignaturas que no tienen la observancia especializada a la hora de presentarlas en las mallas curriculares, entre las cuales figura el Derecho Penitenciario. Es decir, se concluye que el análisis técnico y especializado para ofertar carreras de educación superior en este caso como lo es Derecho, asegura observar y determinar asignaturas que son importantes y requieren su autonomía en su praxis de enseñanza.

La carrera de Derecho en el contexto ecuatoriano, se edifica como importante y trascendental en el compromiso del profesional con la ciudadanía, por tal razón se concluye que la instrucción (pedagogía) del Derecho Penitenciario como asignatura 
autónoma e independiente, corresponde a un compromiso mejor idealizado con la sociedad. De manera que, el estudiante graduado de Derecho que posee este conocimiento ayude a mejorar la calidad de hermandad y comportamiento de la ciudadanía.

\section{FINANCIAMIENTO}

No monetario.

\section{AGRADECIMIENTO}

A la Universidad Regional Autónoma de los Andes, Ibarra, por motivar el desarrollo de la Investigación.

\section{REFERENCIAS CONSULTADAS}

Alpizar, J., y Molina, M. (2017). Las competencias en el contexto de la educación superior. [Competences in the context of higher education]. Atenas, 2(42). 108101. Recuperado de: https://n9.cl/n71an

Asamblea Nacional Constituyente de la República del Ecuador, (2008). Constitución de la República del Ecuador. [Constitution of the Republic of Ecuador]. Montecristi. Registro Oficial 449 de 20-oct-2008. Recuperado de https://n9.cl/sia

Asamblea Nacional de la República del Ecuador (2014) Código Orgánico Integral Penal. [Comprehensive Criminal Organic Code]. Ley 0 Registro Oficial Suplemento 180 de 10-feb.-2014 Ultima modificación: 17-feb.-2021. Quito. CEP. Recuperado de: https://n9.cl/w5nul

Asamblea Nacional del Ecuador. (2010). Ley Orgánica de Educación Superior. [ Organic Law on Higher Education]. Registro Oficial Suplemento 298 de 12-oct.2010. Recuperado de: https://n9.cl/v9bpx

Bonilla García, M. y López Suárez, A. (2016). Ejemplificación del proceso metodológico de la teoría fundamentada. [ Exemplification of the methodological process of grounded theory]. Cinta de Moebio, (57),305-315. Recuperado de: $\underline{\text { https://n9.cl/eoutx }}$ 
Castellanos, R. (2019). Universidad, ciencia y contexto para la calidad académica: Una relación indispensable en los procesos investigativos. [ University, science and context for academic quality: An indispensable relationship in research processes]. Revista Scientific. 2 (4),10-271.Recuperado de: https://n9.cl/d1r29

Cesano, J. D. (2007). Derecho penitenciario: aproximación a sus fundamentos. Principios generales, axiología constitucional y fuentes. [ Penitentiary law: approach to its foundations. General principles, constitutional axiology and sources]. Alveroni Ediciones. Recuperado de: https://n9.cl/ltify

Coca. J. (2007). El sistema penitenciario mexicano: a un paso del colapso. [ The Mexican prison system: one step away from collapse]. IUS. 1 (19),168187.Recuperado de: https://n9.cl/4thax

Durán, M. (2020). Derecho Penitenciario: delimitación de su concepto, función y contenido desde un modelo teleológico-funcional del fin de la pena. [ Penitentiary Law: delimitation of its concept, function and content from a teleological-functional model of the end of the sentence]. Concepc, 88(247), 117-156. Recuperado de: https://n9.cl/9uoof

Duran, M., y Prado, G. (2020). Recomendaciones y propuestas para una reforma penitenciaria. Apuntes para su sistematización y delimitación. [Recommendations and proposals for prison reform. Notes for its systematization and delimitation]. Revista de Derecho de la Pontificia Universidad Católica de Valparaíso. 1(54),151-181.Recuperado de: https://n9.cl/qgvbb

Ferrajoli, L. (2009). Los Fundamentos de los Derechos fundamentales. [The Foundations of Fundamental Rights] TROTTA.

Garzón, A. E., y Romero, Z. (2018). Los modelos pedagógicos y su relación con las concepciones del derecho. [Pedagogical models and their relationship with conceptions of law]. RIDI. 8(2), 311-321.Recuperado de: https://n9.cl/rixx6

Hernández, R., Fernández, C. y Baptista, P. (2014). Metodología de la Investigación. [Research Methodology] MacGRAW-HILL. 
Real Academia de Lengua Española. (2021). Derecho Penitenciario. [ Penitentiary Law] Diccionario panhispánico del español jurídico.Recuperado de: https://n9.cl/co2sv

Silvera, A., Garcés, L. y Pineda, M. (2018). Una reflexión pedagógica del Derecho (parte l): Proyección de competencias y contextos de enseñanza. [A pedagogical reflection of law (part I): Projection of competences and teaching contexts] Espacios, 39(23), 1. Recuperado de: https://n9.cl/1qker

Vargas, J. L. (2016). La legitimación, aplicación y ejecución de la pena en el sistema penal acusatorio. [The legitimation, application and execution of the penalty in the accusatory criminal system] IURIS, 1(15), 61-71. Recuperado de: https://n9.cl/lik5k

Zaffaroni, E. R. (2009). Estructura básica del derecho penal. [ Basic structure of criminal law]. EDIAR 